\title{
SCIENTIFIC WORD, Version 1.0
}

\author{
A SOFTWARE REVIEW ${ }^{1}$
}

\author{
SEMEN KÖKSAL \\ Bradley University \\ Department of Mathematics \\ Peoria, IL 61625
}

\begin{abstract}
Scientific Word is the first fully integrated mathematical word processor in the Windows 3.1 environment, which uses the $\mathrm{T}_{\mathrm{E}} \mathrm{X}$ typesetting language for output. It runs as a Microsoft Windows application program and has two-way interface to $\mathrm{T}_{\mathrm{E}} \mathrm{X}$. The Scientific Word is an object-oriented WYSIWYG word processor for virtually all users who need typesetting scientific books, manuals and papers. It includes automatic equation numbering, spell checking, and $\mathrm{LAT}_{\mathrm{E}} \mathrm{X}$ and DVI previewer.
\end{abstract}

While there are many word processing software packages available today, most do not allow the user freedom to easily manipulate complex mathematical notation. Scientific Word, with its sophisticated typesetting program, called $\mathrm{T}_{\mathrm{E}} \mathrm{X}$, fills this void. It produces high-quality typeset mathematical texts. Its basic interface is similar to other Windows word processors.

After selecting the Scientific Word icon, the main window appears. The Title bar displays the name of the program and the current file; below the Title bar there is the Menu bar, which lists seven menus: File, Edit, Insert, View, Tag, Tools, and Help. Underneath the Menu bar there are two icon bars: the Objects and Tools bars, which contain icons for entering mathematical objects and icons representing special tools. The second bar is the Symbol bar, which contains icons for entering symbols and Greek characters. The left part of the Symbol bar is called the Cache bar. You can fill the Cache bar with icons representing symbols and characters you use most frequently.

\footnotetext{
${ }^{1}$ Received: May, 1993. Revised: August, 1993.
} 
At the bottom of the screen on the Status bar, there are pop item button, item tag popup, section/body tag popup, text tag popup, keyboard popups, fragments popup, and the message area. Since Scientific Word comes with a very well-written user's guide, you can easily learn the functions of these popups and bars. Most of the symbols and characters hidden under the icons can be inserted with a single mouse click. Some of the icons on the Object and Tools bar open a dialog box containing further selections. Headings, bibliographic items, theorems, etc. are accessible via the popups. Although there are keyboard shortcuts for most of the icons, many users will probably prefer to use a mouse. Special symbols, formulas, and characters, or headings, etc. can be embedded in the conventional text and seen on the screen in accordance with WYSIWYG (What You See is What You Get) systems.

Scientific Word commands define the logical structure instead of the appearance. This allows the user to separate the creative process of writing from the mechanical process of layout, an approach known as logical design. However, documents must still be printed in a readable, organized, and visually pleasing format, and Scientific Word ensures such a format. You get most of what you see on the screen. Good use of colors distinguishes the statements. For example, mathematical expressions appear in red, text in black, headings in blue, and theorems and bibliographic items in a gray box. When printed, everything comes in a usual way.

Generally, Scientific Word allows you to preview the document before printing it. However, if there is a major error in the document you cannot print it. Preview shows exactly what you will get. If you cannot get the preview, it means your document has errors. During this process, double clicking on the $\mathrm{T}_{\mathrm{E}} \mathrm{X}$ icon reveals errors. To interpret these errors some familiarity with $\mathrm{T}_{\mathrm{E}} \mathrm{X}$ is required. This can be a difficulty for users who are not familiar with $\mathrm{T}_{\mathrm{E}} \mathrm{X}$. As long as you do not have any errors, you can preview or print your document from within Scientific Word. Consequently, you do not have to deal with $\mathrm{T}_{\mathrm{E}} \mathrm{X}$.

Scientific Word comes with 44 style files for different types of documents (articles, books, letters, memos or reports). These supplemental documents and files are in the "docs" and the "extras" directories. The documents in the "extras" directory include the Scientific Word Technical Reference and documents 
describing some of the supplied $\mathrm{T}_{\mathrm{E}} \mathrm{X}$ styles. The "docs" directory contains shell documents (templates with preset styles) and samples. For example: Letter.tex, a shell document is used for creating letters. To create a letter, open this document, save under another name, and type your letter. Mathart1.tex and Mathart2.tex are shells for creating articles containing mathematics, and in particular, statements of theorems, lemmas, etc. Mitthesi.tex, a shell document is based on the public domain files for the MIT thesis style. It is a great advantage to have these prestyled documents ready for use. If changes are not required, it is easy to create a sophisticated mathematical article. Assuming that no major error has been made during typing, preview and print of the article can be accomplished without any difficulty caused by $\mathrm{T}_{\mathrm{E}} \mathrm{X}$. But if you need a style that is not given by Scientific Word, then you need to go the $\mathrm{LAT}_{\mathrm{E}} \mathrm{X}$ to make necessary changes. This is again another problem for users that are not familiar with $\mathrm{T}_{\mathrm{E}} \mathrm{X}$.

The current version of Scientific Word lacks a search-and-replace function, or a goto-command. It has a macro function, but it does not allow the user to define macros. Scientific Word documents differ from those of $\mathrm{LAT}_{\mathrm{E}} \mathrm{X}$ in the way that they use a minimal set of extra macros provided in "tcilatex.tex". Many of these macros are borrowed from $\mathrm{AMST}_{\mathrm{E}} \mathrm{X}$, and increase the compatibility with $\mathrm{AMST}_{\mathrm{E}} \mathrm{X}$. Hence it is more likely that AMS officials would accept documents in Scientific Word than in LAT $_{\mathrm{E}} \mathrm{X}$. The authors of Scientific Word plan to add $\mathrm{AMST}_{\mathrm{E}} \mathrm{X}$ 's features on top of $\mathrm{LAT}_{\mathrm{E}} \mathrm{X}$ in a future version.

Despite some disadvantages, Scientific Word is one of the most sophisticated and user-friendly word processors for mathematical notation. Its basis is $\operatorname{LAT}_{E} \mathrm{X}$ which is the first system to provide a full set of tools for sectioning, automatic numbering, table of contents, spell checking, automatic formatting, and many other documents constructs. Scientific Word, of course, has all these features. It runs under Windows 3.0 or higher, it has direct access to all the memory in your computer (2MG is required, $4 \mathrm{MG}$ recommended). It is very slow in scrolling through a document, but it is not otherwise significantly slower than other windows-based programs.

Scientific Word does an excellent job by combining an intuitive Windows interface with the power of $\mathrm{LAT}_{\mathrm{E}} \mathrm{X}$. We hope that future versions of Scientific Word will provide more macro and search functions than the current version does, 
and it will be easier to reformat documents and interpret errors during the preview.

Product Reviewed: Scientific Word

TCI Software Research

1190 Foster Road

Las Cruces, NM 88001

1-800-874-2383

(Price $\$ 595.00$, academic discount available). 


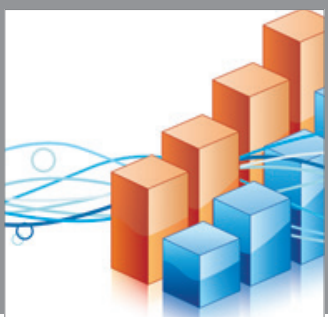

Advances in

Operations Research

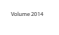

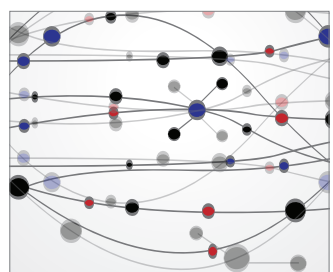

\section{The Scientific} World Journal
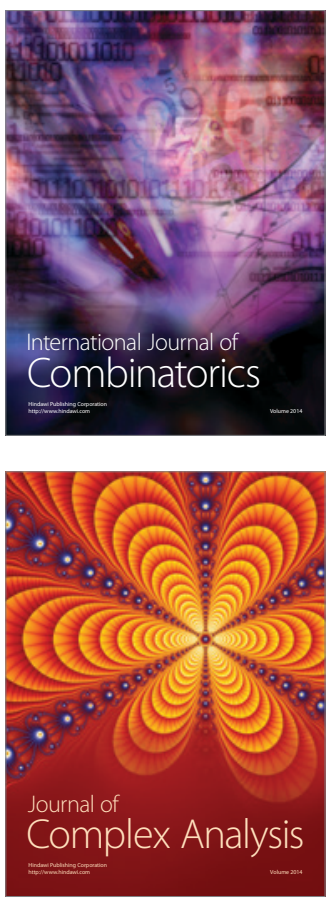

International Journal of

Mathematics and

Mathematical

Sciences
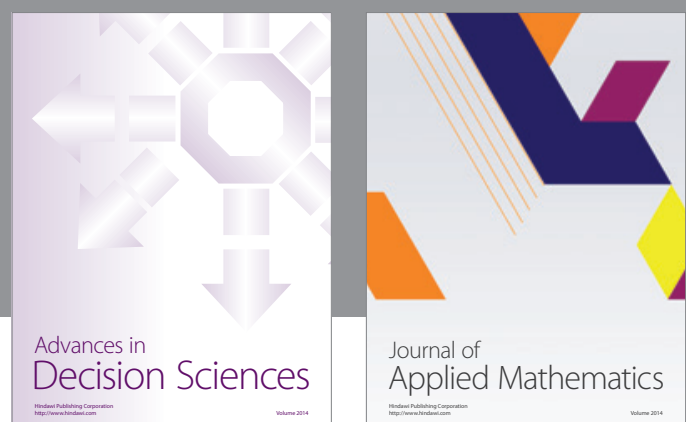

Journal of

Applied Mathematics
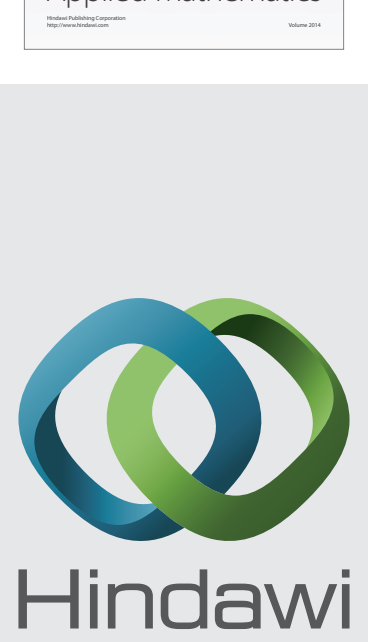

Submit your manuscripts at http://www.hindawi.com
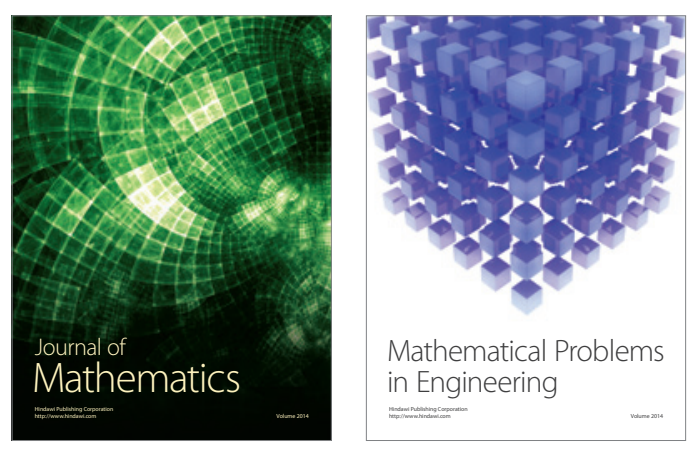

Mathematical Problems in Engineering
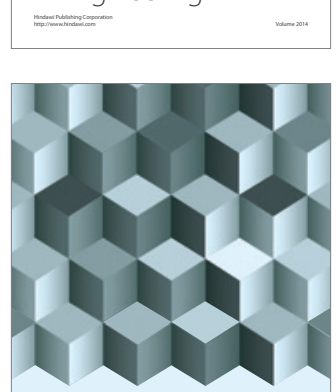

Journal of

Function Spaces
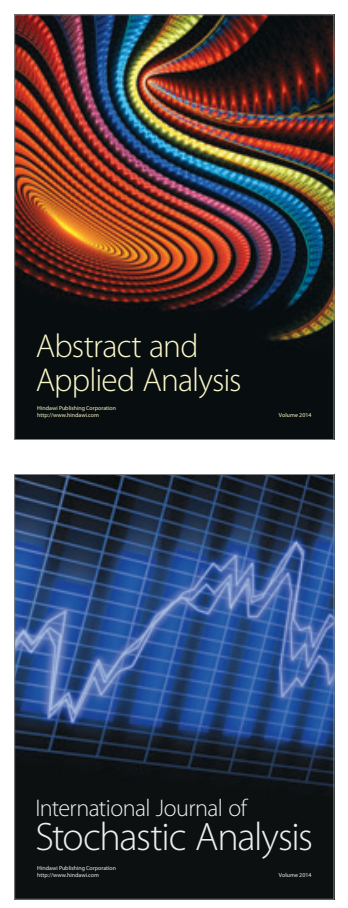

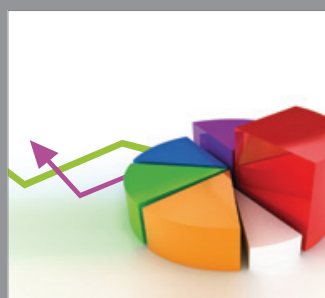

ournal of

Probability and Statistics

Promensencen
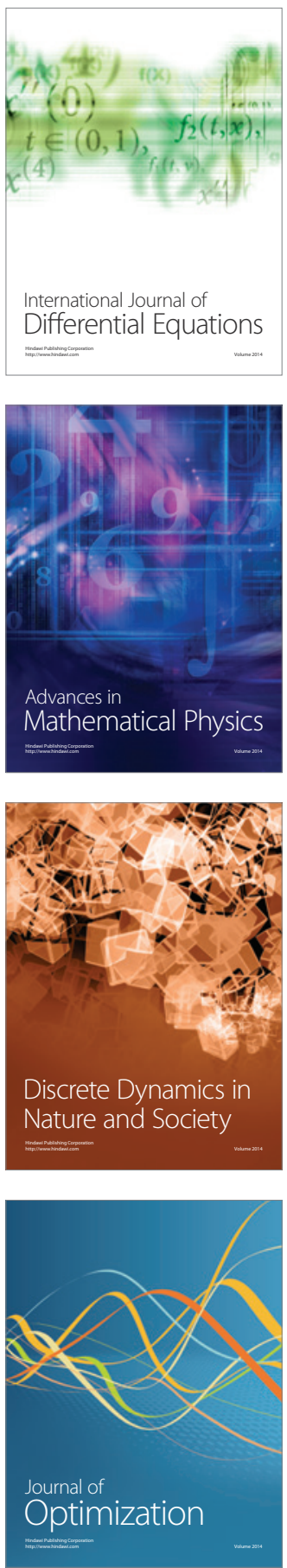